University of Wollongong

Research Online

Faculty of Engineering and Information

Faculty of Engineering and Information

Sciences - Papers: Part A

Sciences

$1-1-2014$

Small field in-air output factors: the role of miniphantom design and dosimeter type

Kirbie Warrener

University Of Wollongong, kjw973@uowmail.edu.au

Benjamin Hug

University Of Western Australia

Paul Liu

University of Sydney

Anna Ralston

Chris O'Brien Lifehouse, Radiation Oncology, Sydney

Martin A. Ebert

University of Wollongong

See next page for additional authors

Follow this and additional works at: https://ro.uow.edu.au/eispapers

Part of the Engineering Commons, and the Science and Technology Studies Commons

Research Online is the open access institutional repository for the University of Wollongong. For further information contact the UOW Library: research-pubs@uow.edu.au 


\title{
Small field in-air output factors: the role of miniphantom design and dosimeter type
}

\begin{abstract}
The commissioning of treatment planning systems and beam modeling requires measured input parameters. The measurement of relative output in-air, Sc is particularly difficult for small fields. The purpose of this study was to investigate the influence of miniphantom design and detector selection on measured Sc values for small fields and to validate the measurements against Monte Carlo simulations. Measurements were performed using brass caps (with sidewalls) or tops (no sidewalls) of varying heights and widths. The performance of two unshielded diodes (60012 and SFD), EBT2 radiochromic film, and a fiber optic dosimeter (FOD) were compared for fields defined by MLCs (5-100 mm) and SRS cones (4-30 $\mathrm{mm})$ on a Varian Novalis linear accelerator. Monte Carlo simulations were performed to theoretically predict Sc as measured by the FOD. For all detectors, Sc agreed to within $1 \%$ for fields larger than $10 \mathrm{~mm}$ and to within $2.3 \%$ for smaller fields. Monte Carlo simulation matched the FOD measurements for all size of cone defined fields to within $0.5 \%$. Miniphantom design is the most important variable for reproducible and accurate measurements of the in-air output ratio, Sc, in small photon fields (less than $30 \mathrm{~mm}$ ). Sidewalls are not required for fields less than or equal too $30 \mathrm{~mm}$ and tops are therefore preferred over the larger caps. Unlike output measurements in water, Scp, the selection of detector type for Sc is not critical, provided the active dosimeter volume is small relative to the field size
\end{abstract}

\section{Keywords}

output, factors, role, miniphantom, air, design, small, dosimeter, type, field

\section{Disciplines}

Engineering | Science and Technology Studies

\section{Publication Details}

Warrener, K., Hug, B., Liu, P., Ralston, A., Ebert, M. A., McKenzie, D. \& Suchowerska, N. (2014). Small field in-air output factors: the role of miniphantom design and dosimeter type. Journal of Medical Physics, 41 021723-021723-8.

\section{Authors}

Kirbie Warrener, Benjamin Hug, Paul Liu, Anna Ralston, Martin A. Ebert, David McKenzie, and Natalka Suchowerska 


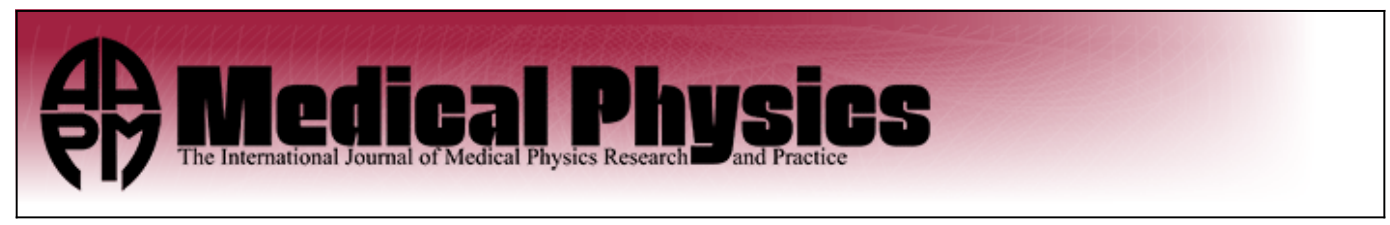

Small field in-air output factors: The role of miniphantom design and dosimeter type

Kirbie Warrener, Benjamin Hug, Paul Liu, Anna Ralston, Martin A. Ebert, David R. McKenzie, and Natalka

Suchowerska

Citation: Medical Physics 41, 021723 (2014); doi: 10.1118/1.4861710

View online: http://dx.doi.org/10.1118/1.4861710

View Table of Contents: http://scitation.aip.org/content/aapm/journal/medphys/41/2?ver=pdfcov

Published by the American Association of Physicists in Medicine

\section{Re-register for Table of Content Alerts}

\section{Create a profile.}




\title{
Small field in-air output factors: The role of miniphantom design and dosimeter type
}

\author{
Kirbie Warrener $\left.{ }^{a}\right)$ \\ Illawarra Cancer Care Centre, Wollongong Hospital, Wollongong, New South Wales 2521, \\ Australia and Centre for Medical Radiation Physics, University of Wollongong, Wollongong, \\ New South Wales 2522, Australia \\ Benjamin Hug \\ School of Physics, University of Western Australia, Crawley, Western Australia 6009, Australia and Department \\ of Radiation Oncology, Sir Charles Gairdner Hospital, Perth, Western Australia 6009, Australia
}

Paul Liu

School of Physics, University of Sydney, Darlington, New South Wales 2008, Australia

Anna Ralston

Chris O'Brien Lifehouse, Radiation Oncology, Sydney, New South Wales 2050, Australia

Martin A. Ebert

School of Physics, University of Western Australia, Crawley, Western Australia 6009, Australia and Department of Radiation Oncology, Sir Charles Gairdner Hospital, Perth, Western Australia 6009, Australia

David R. McKenzie

School of Physics, University of Sydney, Darlington, New South Wales 2008, Australia

Natalka Suchowerska

School of Physics, University of Sydney, Darlington, New South Wales 2008, Australia and Chris O'Brien Lifehouse, Radiation Oncology, Sydney, New South Wales 2050, Australia

(Received 21 June 2013; revised 9 December 2013; accepted for publication 20 December 2013; published 24 January 2014)

Purpose: The commissioning of treatment planning systems and beam modeling requires measured input parameters. The measurement of relative output in-air, $S_{c}$ is particularly difficult for small fields. The purpose of this study was to investigate the influence of miniphantom design and detector selection on measured $S_{c}$ values for small fields and to validate the measurements against Monte Carlo simulations.

Methods: Measurements were performed using brass caps (with sidewalls) or tops (no sidewalls) of varying heights and widths. The performance of two unshielded diodes (60012 and SFD), EBT2 radiochromic film, and a fiber optic dosimeter (FOD) were compared for fields defined by MLCs $(5-100 \mathrm{~mm})$ and SRS cones $(4-30 \mathrm{~mm})$ on a Varian Novalis linear accelerator. Monte Carlo simulations were performed to theoretically predict $S_{c}$ as measured by the FOD.

Results: For all detectors, $S_{c}$ agreed to within $1 \%$ for fields larger than $10 \mathrm{~mm}$ and to within $2.3 \%$ for smaller fields. Monte Carlo simulation matched the FOD measurements for all size of cone defined fields to within $0.5 \%$.

Conclusions: Miniphantom design is the most important variable for reproducible and accurate measurements of the in-air output ratio, $\mathrm{S}_{\mathrm{c}}$, in small photon fields (less than $30 \mathrm{~mm}$ ). Sidewalls are not required for fields $\leq 30 \mathrm{~mm}$ and tops are therefore preferred over the larger caps. Unlike output measurements in water, $S_{c p}$, the selection of detector type for $S_{c}$ is not critical, provided the active dosimeter volume is small relative to the field size. () 2014 American Association of Physicists in Medicine. [http://dx.doi.org/10.1118/1.4861710]

Key words: fibre optic dosimeter, diode, film, miniphantom, $\mathrm{S}_{\mathrm{c}}$, in-air output ratio

\section{INTRODUCTION}

Stereotactic radiosurgery (SRS) and intensity modulated radiation therapy (IMRT) have led to the increased use of radiotherapy fields with dimensions as small as $4 \mathrm{~mm}$. In such small fields, dosimetry is complicated by both the beam characteristics and the detector design. The three conditions that are commonly used to classify a field as being small are when there is loss of lateral charged particle equilibrium, when there is partial blocking of the photon source by the collimators or when volume averaging across the detector is relatively large. ${ }^{1}$ For $6 \mathrm{MV}$ photon beams from medical accelerators this generally occurs below $30 \mathrm{~mm}$.

It is conventional to separate the in-water output ratio, $S_{c p}$, into its components arising from changing head scatter conditions and phantom scatter: ${ }^{2}$

$$
S_{c p}(s) \approx S_{c}(s) \times S_{p}(s),
$$


where $S_{c}$ is the in-air output ratio, $S_{p}$ is the phantom scatter factor, and $s$ is the field size at isocenter.

$\mathrm{S}_{\mathrm{c}}$ has historically been considered a "head scatter factor," where the change in head scatter conditions affected the dose reaching the point of measurement at the isocenter. However, there are at least two other contributing factors to a variation in $S_{c}$ : the backscatter to the Linac ionization chamber and the occlusion of the primary beam.

The component arising from the changing head scatter conditions has been described by a number of terms including "head scatter factor," "collimator scatter factor," and "in-air output factor." In this paper, this quantity will be referred to as the in-air output ratio, $\mathrm{S}_{\mathrm{c}}{ }^{3}$ The in-air output ratio characterizes the photon fluence at a point in a therapeutic radiation beam as a function of field size. $S_{c}$ is defined as the ratio of primary collision water kerma in free space, $K_{p}$, per monitor unit between the collimator setting of interest, $\mathrm{c}$, and a reference collimator setting, $c_{\text {ref }}$, (typically $100 \times 100 \mathrm{~mm}^{2}$ ) at the same point,

$$
S_{c}(c) \equiv \frac{K_{p}\left(c ; z_{r e f}\right) / \mathrm{MU}}{K_{p}\left(c_{r e f} ; z_{r e f}\right) / \mathrm{MU}},
$$

where $\mathrm{z}_{\text {ref }}$ is the source-to-detector distance. ${ }^{3}$

Measured in-air output ratios are useful in beam modeling and dose calculations and it is recommended they be part of the commissioning process for each linear accelerator. ${ }^{1}$ Zhu et $a .^{3}$ state that a reasonable estimation of $\mathrm{S}_{\mathrm{c}}$ can be obtained by taking a ratio of ionization readings with a detector placed inside a miniphantom with sufficient thickness to eliminate electron contamination. For small fields below $30 \mathrm{~mm}$ width, there are few studies reporting values of $S_{c}$ either from measurements ${ }^{4-6}$ or Monte Carlo simulations. ${ }^{7}$ The smallest field for which $S_{c}$ has been successfully measured and reported has been $10 \mathrm{~mm}$ (Refs. 6 and 8) and for Monte Carlo $5 \mathrm{~mm}^{7}$

\section{A. Miniphantoms}

Various build-up caps and miniphantom designs have been used for measurement of $S_{c}$ in small fields. ${ }^{4,5,8}$ The AAPM TG-74 (Ref. 3) and IPEM Report 103 (Ref. 1) recommend the use of a water-equivalent miniphantom for field sizes larger than $50 \mathrm{~mm}$ and a miniphantom made of a high-density material such as brass for field sizes below $50 \mathrm{~mm}$. Normalizing the ratio obtained with the high-density miniphantom to an intermediate field size $(30-50 \mathrm{~mm})$ and multiplying the resultant values by the $S_{c}$ measured for the intermediate field size with a water-equivalent miniphantom will give values for $\mathrm{S}_{\mathrm{c}}$ with very little error. ${ }^{3}$ This approach has been colloquially termed "daisy chaining."

There are four important considerations in miniphantom design: material composition, height, thickness of the sidewalls and overall width. It has been shown that measurement of $S_{c}$ is not dependent on the atomic number of high-density miniphantoms in field sizes with dimensions less than $40 \mathrm{~mm}$ in a $6 \mathrm{MV}$ photon beam. ${ }^{4}$ However, at larger field sizes and higher energies this is not the case due to the change in energy spectrum with field size.
The AAPM TG-74 (Ref. 3) argues that a miniphantom height equivalent to the depth of dose maximum $\left(\mathrm{d}_{\max }\right)$ is insufficient to eliminate electron contamination and a waterequivalent height of $100 \mathrm{~mm}\left(\mathrm{~d}_{100}\right)$ is recommended, based on measurements of the electron contamination in a $24 \mathrm{MV}$ photon beam..$^{10}$ If electron contamination is not eliminated, the detector reading will erroneously increase, particularly at larger field sizes. ${ }^{3}$ There is no recommendation for the more commonly used $6 \mathrm{MV}$ photon beam.

The AAPM TG-74 (Ref. 3) also recommends that the lateral dimensions of the miniphantom should be large enough to maintain lateral charged particle equilibrium (CPE), approximately $1.3 \mathrm{~g} / \mathrm{cm}^{2}$ for a $6 \mathrm{MV}$ photon beam ${ }^{11}$ unless experimental verification shows a thinner wall is sufficient for local conditions. Li et al. ${ }^{11}$ showed that the error introduced into the value of $S_{c}$ due to a break down in lateral CPE is negligible and that the sidewall thickness of the miniphantom can be reduced below that required to achieve lateral CPE, provided that they are thick enough to prevent the effect of electron contamination. McKerracher and Thwaites ${ }^{6}$ have concluded that lateral electron contamination is small enough that no sidewalls are required for a $6 \mathrm{MV}$ photon beam in field sizes below $40 \mathrm{~mm}$. An equivalent height of $d_{\max }$ is sufficient for this energy and range of field size.

In small fields, the width of the miniphantom must also be smaller than the high dose region (dose $\geq 99 \%$ ) of the radiation field, so that the scatter generated in the miniphantom is constant across the range of fields measured. This necessarily means that for very small fields in clinical use $(4 \mathrm{~mm})$, even when using a high density miniphantom, it will be too large to maintain lateral CPE.

Therefore, the ideal characteristics of an ideal miniphantom are (a) to be physically smaller than the high dose region, (b) to be thick enough to remove contamination electrons, and (c) able to be positioned accurately and reproducibly.

Detectors used in measurements of $S_{c}$ in small fields need to have a small sensitive volume, to minimize volume averaging effects, and high sensitivity, to keep noise to an acceptable level. Thus, there is a trade-off between a sensitive volume small enough to achieve good spatial resolution and large enough to provide good signal to noise ratio.

The dimensions of the material surrounding the sensitive volume, i.e. the detector housing also limits the minimum field size for which $S_{c}$ can be accurately measured. Ideally the width of the detector housing should be smaller than the high dose region of the radiation field so that the scatter generated in the housing is constant for all field sizes under consideration. One way to achieve this is to take measurements at an extended source-to-surface distance (SSD). However, this is not recommended due to the different projections of the flattening filter onto the detector at various SSDs for the same collimator. ${ }^{1,12}$

The three types of commercially available detectors that have commonly been used for small field dosimetry are small volume ionization chambers, diamond detectors, and unshielded silicon diodes. Ionization chambers and diamond detectors typically have sensitive volumes that are too wide 
for the smallest clinical fields. Diode detectors can have small sensitive volumes; however, their housings are typically 4-7 mm wide. Radiochromic film has a high spatial resolution and plastic scintillation detectors have small active volumes with minimal housing, ostensibly making them both suitable detectors for use in small fields.

The aim of this study is to investigate experimental factors affecting the measurement of $\mathrm{S}_{\mathrm{c}}$ for small fields, specifically the influence of miniphantom design features and detector selection. The measurements are compared to Monte Carlo simulations. The novelty of this study is that it reports the value of $\mathrm{S}_{\mathrm{c}}$ for a clinical beam, derived using Monte Carlo simulations and measured values, taken with a range of detectors and miniphantom designs for fields as small as $4 \mathrm{~mm}$. These results will provide indicators of how to best measure $S_{c}$ in challenging small field conditions.

\section{MATERIALS AND METHODS}

\section{A. Experimental design}

Measurements were performed using the $6 \mathrm{MV}$ SRS mode (1000 MU/min; SRS flattening filter) on a Varian Novalis Tx linear accelerator (Varian Oncology Systems, Palo Alto). Collimation was achieved using Varian high-definition (HD120) MLCs and BrainLab stereotactic cones. The manufacturers' specified effective point of measurement of each detector was positioned at isocenter (1000 $\mathrm{mm}$ from the focal spot).

Two miniphantom designs (caps and tops) were used (Fig. 1). All miniphantoms were made from common brass (density $8.4 \mathrm{~g} / \mathrm{cm}^{3}$ ) with an equivalent height of either $\mathrm{d}_{\max }$ ( $2.2 \mathrm{~mm}$ brass) or $\mathrm{d}_{100}$ (15 mm brass) in water. The caps have a physical diameter of $11 \mathrm{~mm}$ and sidewall equivalent thickness of $d_{\max }$. The height of the cap miniphantom is sufficiently large to surround both the sensitive volume and the housing for the diodes. The tops have zero sidewall thickness and physical diameters of 3, 5, and $7 \mathrm{~mm}$ (Fig. 1).

Table I summarizes the detectors used and their sensitive volumes and housing dimensions.

The $0.125 \mathrm{~cm}^{3}$ PTW Semiflex ionization chamber is recommended for use in fields of widths $20-400 \mathrm{~mm}$. The PTW Diode E (60012) and the IBA stereotactic field diode (SFD) are both unshielded p-type diodes specifically designed for use in small photon fields. For the above dosimeters, measurements were made with a PTW Unidos E electrometer.

The air-core fiber optic dosimeter (FOD) is a plastic scintillation detector developed for applications in megavoltage photon dosimetry where high spatial resolution is required. ${ }^{13}$ The detector makes use of an air core light guide to trans-

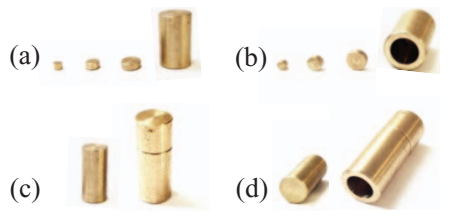

FIG. 1. The brass top and cap miniphantom designs used in this study. (a) and (b) 3, 5, and $7 \mathrm{~mm}$ diameter $\mathrm{d}_{\max }$ tops and $11 \mathrm{~mm}$ diameter $\mathrm{d}_{\max }$ cap and, (c) and (d) $7 \mathrm{~mm}$ diameter $\mathrm{d}_{100}$ top and $11 \mathrm{~mm}$ diameter $\mathrm{d}_{100}$ cap.
TABLE I. The external dimension and sensitive volume dimensions of each detector used in this study.

\begin{tabular}{|c|c|c|c|c|}
\hline \multirow[b]{2}{*}{ Detector } & \multicolumn{3}{|c|}{ Sensitive volume } & \multirow{2}{*}{$\begin{array}{c}\text { External } \\
\text { dimension } \\
(\mathrm{mm})\end{array}$} \\
\hline & $\begin{array}{l}\text { Diameter } \\
(\mathrm{mm})\end{array}$ & $\begin{array}{l}\text { Thickness or } \\
\text { length }(\mathrm{mm})\end{array}$ & $\begin{array}{l}\text { Volume } \\
\left(\mathrm{mm}^{3}\right)\end{array}$ & \\
\hline $\begin{array}{l}\text { PTW Semiflex } \\
\text { Chamber (IC) }\end{array}$ & 5.5 & 6.5 & 125 & 6.2 \\
\hline PTW diode E (60012) & 1.12 & 0.0025 & 0.0025 & 7 \\
\hline $\begin{array}{l}\text { IBA stereotactic diode } \\
\text { (SFD) }\end{array}$ & 0.6 & 0.06 & 0.017 & 4 \\
\hline $\begin{array}{l}\text { Fiber optic dosimeter } \\
\text { (FOD) }\end{array}$ & 1 & 1 & 0.8 & 2 \\
\hline $\begin{array}{l}\text { Gafchromic EBT2 } \\
\text { film }\end{array}$ & $\ldots$ & 0.028 & $\ldots$ & $\ldots$ \\
\hline
\end{tabular}

port the scintillation signal out of the primary radiation field, avoiding the generation of a Cerenkov background signal. The FOD system has a rapid response and good dosimetric performance. ${ }^{13-15}$ The photomultiplier detector system used in this study is described in detail by Liu et al. ${ }^{16}$

$\mathrm{S}_{\mathrm{c}}$ was measured with the ionization chamber, diodes, and FOD in an empty PTW MP3 motorized tank which has a positional accuracy of $0.1 \mathrm{~mm}$. The ionization chamber, diodes, and FOD were supported by Styrofoam ${ }^{\mathrm{TM}}$ (Dow) (density $0.03 \mathrm{~g} / \mathrm{cm}^{3}$ ), to increase the distance between the point of measurement and the metal scanning arm of the water tank, whilst ensuring a rigid and reproducible setup. To confirm that the tank and foam did not contribute excess scatter, measurements were taken under "scatter free" conditions, essentially at a point in air, supporting the detector on a minimal foam extension rod, removing all scattering bodies including the water tank. Differences between the two setups were less than $0.3 \%$. The diode detectors were oriented with their stem axis parallel to the beam axis, whereas the FOD was oriented with its stem axis perpendicular to the beam axis.

The diodes and the FOD were centered in the radiation field by scanning in $0.1 \mathrm{~mm}$ increments to find the point of maximum reading. This was performed at the smallest field size setting for each collimation method $(4 \mathrm{~mm}$ diameter cone, $5 \mathrm{~mm}$ MLC field size). The square MLC field sizes used were $5,10,20,30,40,60,80$, and $100 \mathrm{~mm}$ with jaw settings as recommended by the vendor $(8,12,22,32,42,60,80$, and $100 \mathrm{~mm}$, respectively). The cone diameters used were 4, 7.5, $10,12.5,15,20,25$, and $30 \mathrm{~mm}$ with the jaws kept constant at $50 \times 50 \mathrm{~mm}^{2}$.

Gafchromic EBT2 film is a self-developing radiochromic film, designed for the measurement of absorbed dose of highenergy photons. EBT2 film pieces $\left(50 \times 50 \mathrm{~mm}^{2}\right)$ were positioned perpendicular to the beam axis in-air by placing them over a holder, the central section of which was cut out (45 $\mathrm{mm}$ internal diameter). This holder was extended $300 \mathrm{~mm}$ off the end of the treatment couch to minimize scatter. Each sheet of film used had its own calibration set. These calibration films were exposed at $15 \mathrm{~mm}$ depth in solid water 
at field size $100 \times 100 \mathrm{~mm}^{2}$ in a $6 \mathrm{MV}$ photon beam at $1000 \mathrm{~mm}$ SSD. A minimum of 10 separate films were exposed for each cone. To minimize the uncertainty in conversion from optical density to dose, for each field size the monitor units were scaled up by the inverse of the nominal cone output factors, such that approximately the same dose was delivered to each film piece. The measured dose values were then scaled down by the same nominal factors. Films were kept in a low-light environment for $48 \mathrm{~h}$ after exposure before being scanned with an EPSON 10000 XL scanner. The scanner was used in transmission mode with $150 \mathrm{dpi}$ ( $0.17 \mathrm{~mm}$ pixel size) and 48 bit color (16 bit per channel) RGB with all corrections turned off. Consistent film orientation was maintained (portrait). All films were scanned individually, using only the central portion of the scanner, eliminating the known off-axis nonuniformity response ${ }^{17}$ and only the red channel data were used. ${ }^{18}$ A circular region of interest (ROI) in Image $\mathbf{J}$ was used to determine the mean pixel value in the high dose region of field. The ROI was $1 \mathrm{~mm}$ in diameter to approximate the size of the sensitive volumes of the other detectors used in the study.

During exposure, the selected miniphantom was placed over the sensitive volume of the detector using the overhead lasers. Winston-Lutz positioning tests confirmed the coincidence between the radiation isocenter and the overhead lasers to within $0.3 \mathrm{~mm}$.

The cap and top miniphantoms were used with the 60012 diode to assess the sidewall effects. Electron contamination removal was evaluated using miniphantoms with two equivalent heights, $d_{\max }$ and $d_{100}$, and a range of top widths. The top width measurements were also made using the PTW Semiflex ionization chamber. $S_{c}$ was then determined with the 60012 diode, SFD diode, FOD, and EBT2 film with a selected miniphantom, to compare detector performance. All $S_{c}$ results were normalized at the $30 \mathrm{~mm}$ field size. This field size was chosen as the intermediate field size where the detector/miniphantom combinations were considered to perform accurately.

\section{B. Monte Carlo simulations}

Monte Carlo (MC) simulations of a Varian Novalis Tx linear accelerator treatment head were performed using the BEAMnrc MC code package. The geometry and composition of head components was provided in confidence by Varian. The accelerator was modeled operating in SRS mode, with a smaller flattening filter than is used in normal mode, with the default jaw-defined field size of $50 \times 50 \mathrm{~mm}^{2}$. The photon and electron transport cut-offs (PCUT and ECUT) were $0.01 \mathrm{MeV}$ and $0.521 \mathrm{MeV}$, respectively. A scoring plane was defined following the stereotactic cone collimators at a distance of $800 \mathrm{~mm}$ and phase-space files produced for $4,7.5$, $10,12.5,15,20$, and $30 \mathrm{~mm}$ cone diameters. Phase-space files contain information about the particle characteristic at the scoring plane, including a latch variable describing each particle's interaction history. ${ }^{19}$ The number of particles in each of the phase-space files ranged from 23521 for the $4 \mathrm{~mm}$ cone to 616371 for the $30 \mathrm{~mm}$ cone diameter.

To validate the MC model, the produced phase-space files were used as inputs into a DOSXYZnrc (Refs. 20 and 21) Monte Carlo dose calculation. The dose was calculated in a water phantom with an SSD of $1000 \mathrm{~mm}$ and compared to beam profile measurements made with EBT2 Gafchromic film for all cone sizes. The energy transport cut-offs, PCUT and ECUT, were set to 0.01 and $0.521 \mathrm{MeV}$, respectively. The dose was calculated in the water phantom for all field sizes and beam profiles compared at phantom depths ranging from the surface to $200 \mathrm{~mm}$. The characteristics of the electron beam from the guide were adjusted in the BEAMnrc simulation to achieve the best match possible between measured and simulated beam profiles for all cone diameters at depths ranging from the surface to $200 \mathrm{~mm}$. BEAMnrc source number 19 with a focal spot size of $1.3 \mathrm{~mm}$ and an electron kinetic energy of $6.2 \mathrm{MeV}$ ultimately gave the best fit. The term "characteristics" refers to the focal spot size and the initial electron energy. With the geometry used and selected initial settings for the beam, the model fitting was remarkably good. Therefore little tuning was needed, apart from small changes to spot size and beam energy.

To simulate the FOD detector the Geant4 Monte Carlo toolkit was used. ${ }^{22}$ The FOD active volume was defined as a cylinder with a diameter and height of $1 \mathrm{~mm}$ consisting of Bicron BC400 scintillator material (density $1.03 \mathrm{~g} / \mathrm{cm}^{3}$ ). The cylindrical brass build-up cap was defined as common brass (63\% Copper, $37 \%$ Zinc, density $8.55 \mathrm{~g} / \mathrm{cm}^{3}$ ) with a diameter of $3 \mathrm{~mm}$ and a height of $2.3 \mathrm{~mm}$. The center of the sensitive detector volume was positioned at the center of the simulation world volume with the brass build-up cap positioned directly on top of the detector volume. The default step size was set to $0.2 \mathrm{~mm}$ for all particle types and the secondary production lower energy cut was set to $250 \mathrm{eV}$. The physics processes were defined using a user specified physics list and included photoelectric effect, Compton scatter and gamma conversion for photons, electron multiple scattering, ionization, bremsstrahlung production, and electron annihilation for positrons and electrons.

The phase-space files that were produced using BEAMnrc were imported using a MATLAB (Mathworks, Natick) function and exported as a binary file in a format containing each particle's type, energy, position, and direction cosines, which were then fired one by one at the detector geometry using Geant4's PrimaryGeneratorAction class. The distance between the center of the detector active volume and the phasespace file plane, where each particle in the phase space file was reused in the Geant 4 simulation, was $200 \mathrm{~mm}$ for all simulations reflecting the detector effective point of measurement being positioned at isocenter. The energy deposited in the scintillating material was recorded. Ten simulations for each cone diameter were completed using varying seed numbers and the total energy deposited within the detector volume averaged and a standard deviation for the ten simulations calculated. To calculate the $S_{c}$ the energy deposited in the detector volume for each cone size was normalized to the number of electrons incident on the target in the BEAMnrc simulation 
and the recycle number, which corresponds to the number of times each particle is fired within the Geant 4 simulation. $S_{c}$ was then calculated as the ratio of the normalized energy per incident electron for the respective cone size to that calculated for the $30 \mathrm{~mm}$ cone size.

The theoretical $\mathrm{S}_{\mathrm{c}}$ was calculated by determining the KERMA from the spectra for each cone. The ratio of the KERMA in air gives the theoretical Sc.

\section{RESULTS AND DISCUSSION}

The effect of top and cap miniphantom design on the measured $S_{c}$ is shown in Fig. 2. For field sizes from 20 to $100 \mathrm{~mm}$, the measured output factors for all designs agree to within $0.3 \%$. McKerracher and Thwaites ${ }^{8}$ found a similar agreement between in-air output measurements for tops and caps with equivalent heights of $\mathrm{d}_{\max }$ and $\mathrm{d}_{50}$ in a $6 \mathrm{MV}$ photon beam. However, for fields smaller than $20 \mathrm{~mm}$, the measured $S_{c}$ is critically dependent upon the miniphantom design. There is a feature of approximately $0.2 \%$ for the $10 \mathrm{~mm}$ cone that appears not to align with a visual extrapolation of the line. We do not speculate on the origin of this feature. If the high dose region of the field becomes smaller than the miniphantom, the $S_{c}$ for these fields will appear to be lower, as the scatter generated in the miniphantom will not be constant with field size relative to the normalization field size. This effect will be termed loss of phantom scatter constancy and is more significant for the caps, because they are physically wider. Therefore, tops were used for all subsequent measurements.

Figure 2 also depicts the effect of miniphantom height. As the field size decreases, the fall in $S_{c}$ is greater when the miniphantom height is increased (from $d_{\max }$ to $d_{100}$ ). This may be explained by two phenomena. First, in very small fields beam divergence causes a greater proportion of scattering material to lie outside the high dose region of the field for tall miniphantoms. Second, there is a greater difficulty in aligning a tall and narrow miniphantom with the beam axis, increasing the uncertainty in measurement. For these reasons tops of height $d_{\max }$ were used for all subsequent measurements.

The effect of varying the width of the tops on the measured $S_{c}$ is shown for MLCs and cones in Figs. 3(a) and 3(b), respectively. For MLC fields larger than $30 \mathrm{~mm}, \mathrm{~S}_{\mathrm{c}}$ for the diode with the $3 \mathrm{~mm}$ wide top deviates significantly from that measured with the ionization chamber and diode with wider tops. This is interpreted as the effect of insufficient lateral shielding, as removing sidewalls means that side scatter associated with the larger fields is able to reach the sensitive volume of the detector. ${ }^{3}$ Figure 3 shows that between 10 and $30 \mathrm{~mm}$ the measured $\mathrm{S}_{\mathrm{c}}$ with all miniphantom tops agree to within $1 \%$. For fields smaller than $10 \mathrm{~mm}, \mathrm{~S}_{\mathrm{c}}$ decreases with an increase in the top width [Figs. 3(a) and 3(b)], due to a loss of phantom scatter constancy as defined above. Even a $3 \mathrm{~mm}$ top will be wider than the high dose region of the $4 \mathrm{~mm}$ field. However attempts to further reduce the top width to $2 \mathrm{~mm}$ were unreliable, because the uncertainty of positioning is too great for the results to be of value. From the results shown in Figs. 3(a) and 3(b), the $3 \mathrm{~mm}$ top was considered to be the best compromise to measure $S_{c}$ in small fields and was used for all subsequent measurements.

The experimental and Monte Carlo values of $\mathrm{S}_{\mathrm{c}}$, derived as described in the methods section, agreed to within $2.3 \%$ at the smallest field size (Fig. 4). Radiochromic film measurements of $\mathrm{S}_{\mathrm{c}}$ agree with the FOD measurements to within $0.5 \%$, except for the smallest field size of $4 \mathrm{~mm}$. At this field

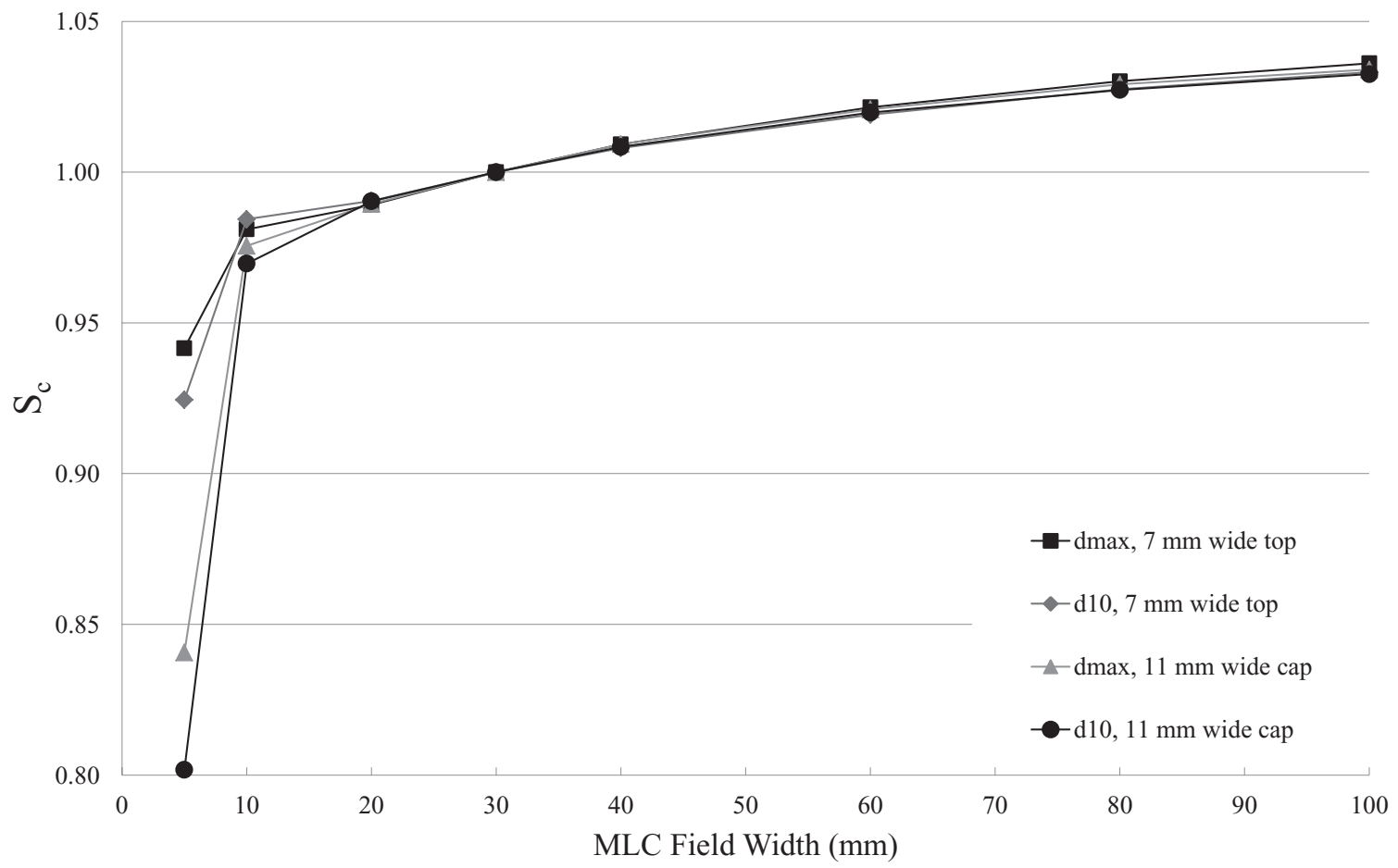

FIG. 2. $\mathrm{S}_{\mathrm{c}}$ for MLCs measured using 60012 diode with $11 \mathrm{~mm}$ wide caps and $7 \mathrm{~mm}$ wide tops. The equivalent heights of the caps and tops are either $\mathrm{d}_{\mathrm{max}}$ or $\mathrm{d}_{100}$. The uncertainty bars are smaller than the symbols used. 


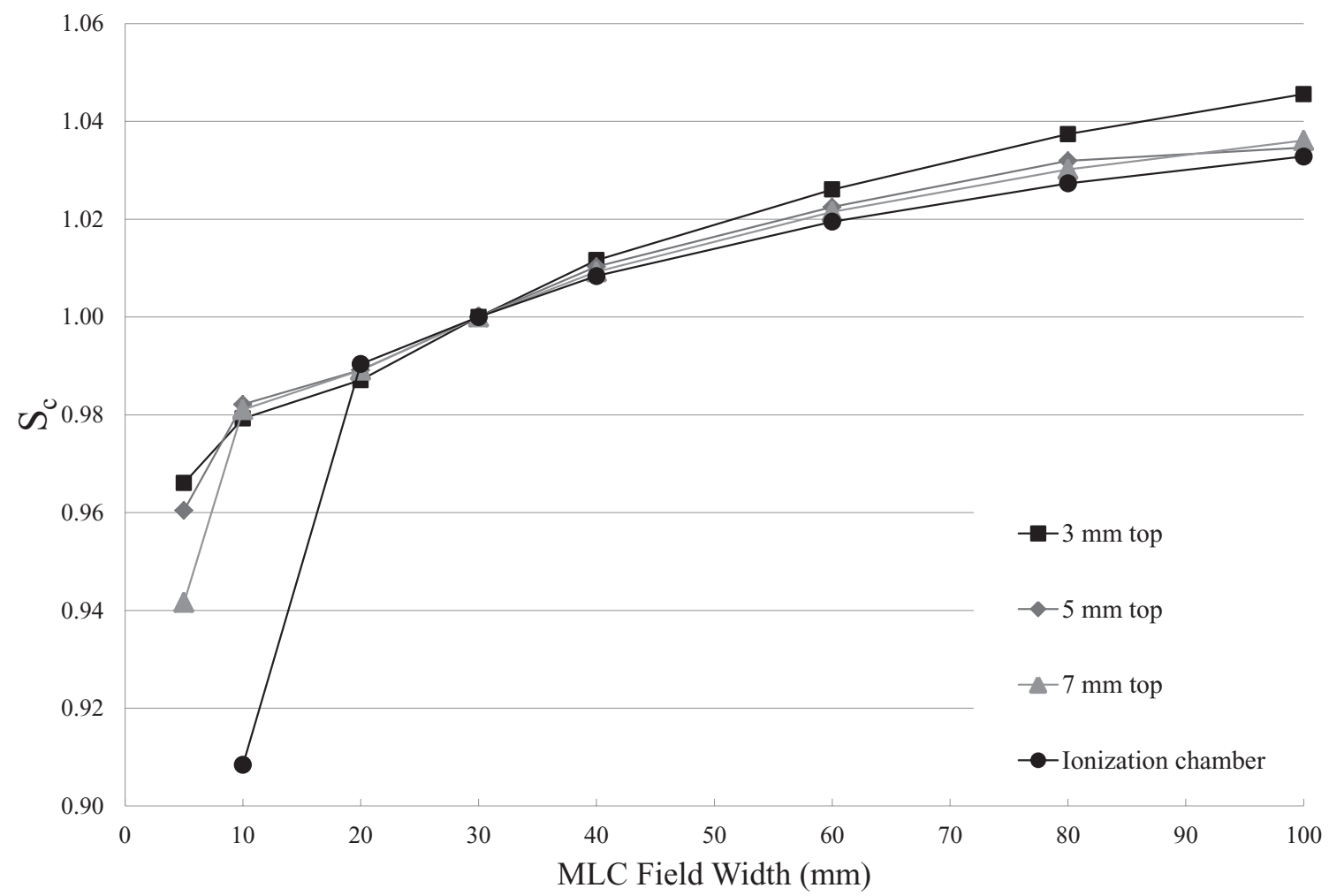

(a)

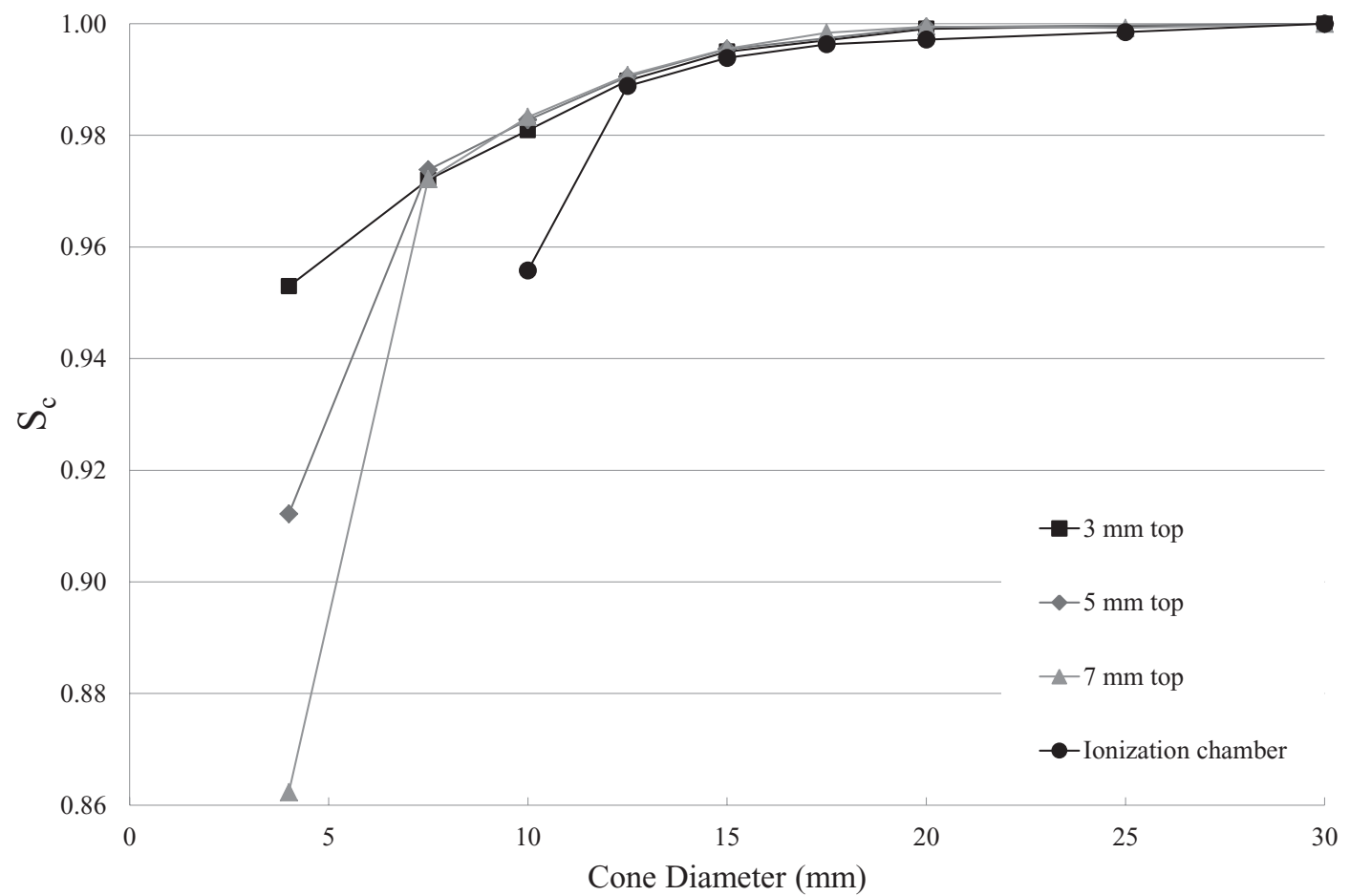

(b)

FIG. 3. $\mathrm{S}_{\mathrm{c}}$ for (a) MLCs and (b) cone collimation measured using 60012 diode with tops of width 3,5 , and $7 \mathrm{~mm}$ and an ionization chamber with $11 \mathrm{~mm}$ cap. The equivalent heights of the tops and cap are $\mathrm{d}_{\max }$. The uncertainty bars are smaller than the symbols used. 


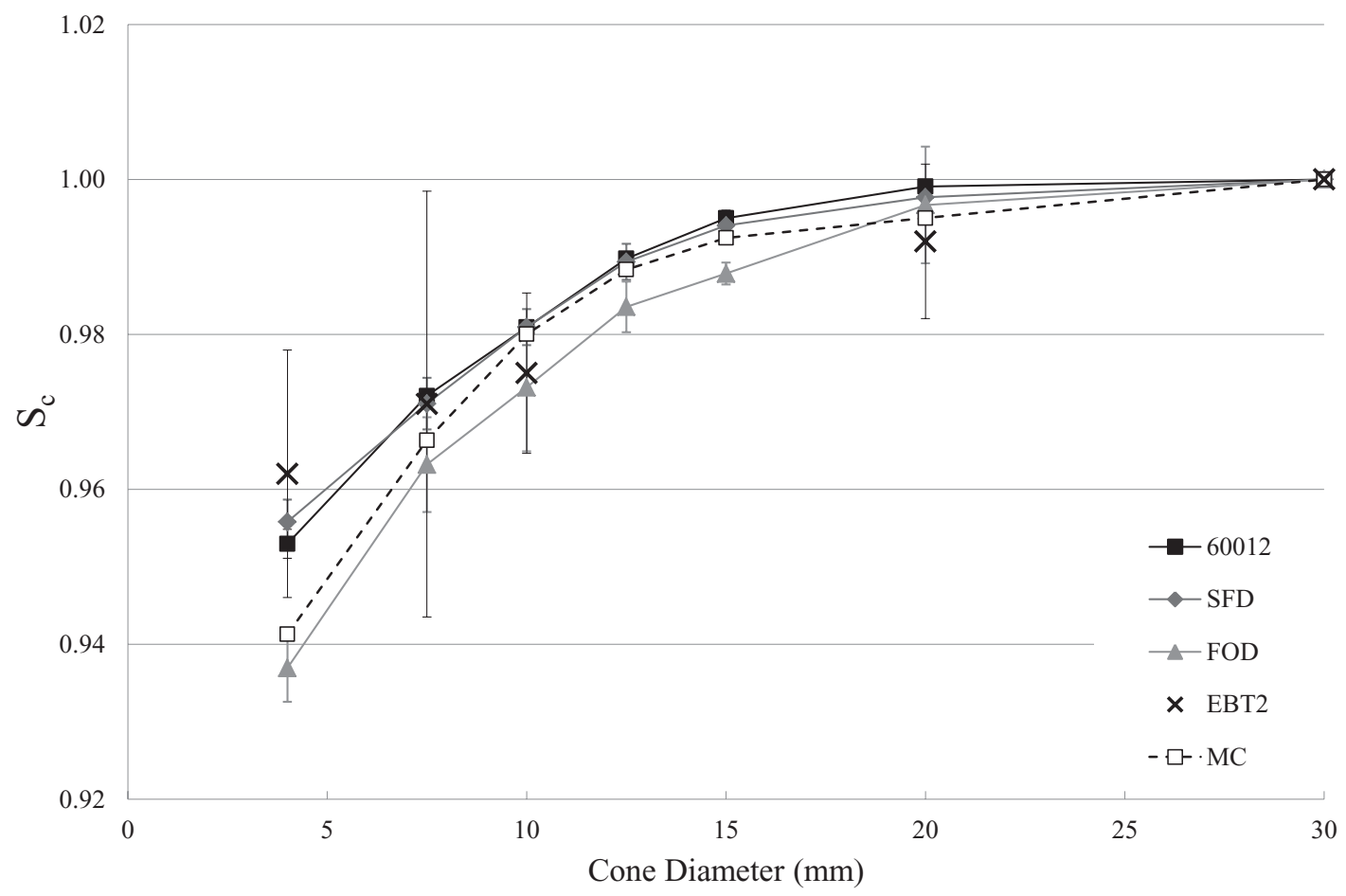

FIG. 4. $\mathrm{S}_{\mathrm{c}}$ for cone collimation measured using the 60012 diode, SFD diode, FOD and EBT2 film with 3 mm wide tops with equivalent height $\mathrm{d}_{\mathrm{max}}$. Monte Carlo calculated $\mathrm{S}_{\mathrm{c}}$ are also shown. The uncertainty bars represent one standard deviation.

size the Monte Carlo derived values of $\mathrm{S}_{\mathrm{c}}$ for the dosimeter most closely match those of the FOD (0.5\% difference). Between 10 and $15 \mathrm{~mm}$ there is a $0.8 \%$ difference and the shapes of the curves appear different. The theoretical value of $\mathrm{S}_{\mathrm{c}}$ calculated from the ratio of KERMA for each cone at a point in air overlay the measured FOD values of $S_{c}$ to within $0.5 \%$.

The uncertainty bars in this study represent one standard deviation for multiple measurement sessions. The number of measurement sessions for each dosimeter ranged from three, for the diodes and FOD, up to at least five for the Gafchromic EBT2 film as it had the highest variability of the dosimeters employed. Within each measurement session a minimum of three readings were taken for each data point. The uncertainty bars represent Type A uncertainties ${ }^{23}$ and include detector reproducibility, setup variation between separate measurement sessions and variation in beam characteristics. The Type A uncertainties were within $1.4 \%$ for the FOD, $0.2 \%$ for the 60012 and $0.3 \%$ for the SFD for all field sizes. The uncertainty in the EBT2 film was determined to be within $2.0 \%$ (1 SD) and is consistent with that reported in the literature. ${ }^{17}$ Although the overall maximum difference in $S_{c}$ is $2.3 \%$ for the $4 \mathrm{~mm}$ cone, this is acceptable given the difficulty of the measurements. The agreement between the $S_{c}$ measurements obtained using EBT2 film, the FOD and the diodes, with their relative densities of $1.30,1.03$, and $2.33 \mathrm{~g} / \mathrm{cm}^{3}$, respectively, shows that the dosimetric water equivalence of a detector's sensitive volume over this range is not a consideration in the measurement of $S_{c}$. This is in contrast to in-water output factors, $S_{c p}$, where the density of the detector affects the phantom scatter component $^{24}$ and correction factors are required. ${ }^{25}$
From the Monte Carlo simulation, we determined that almost the entire dose deposited in the FOD scintillator arises from primary radiation ( $>99 \%$ for the $30 \mathrm{~mm}$ cone), not scattered radiation (from the flattening filter or other components in the Linac head). This is reflected in the measured data in Fig. 3, which shows that between 10 and $30 \mathrm{~mm}$ the measured $\mathrm{S}_{\mathrm{c}}$ with all miniphantom tops of widths 3,5 , and $7 \mathrm{~mm}$, agree to within $1 \%$, confirming that below $30 \mathrm{~mm}$ there must be minimal contribution to the dose at the detector from lateral scatter. Cap design miniphantoms are therefore not necessary in small fields and may even lead to errors stemming from loss of phantom scatter constancy.

Many treatment planning systems use a combination of $S_{c}$ and $S_{p}$ to model the scattering conditions within a patient or phantom. $S_{\mathrm{p}}$ is usually determined by dividing the known total scatter factor $S_{\mathrm{cp}}$ by the measured value of $S_{\mathrm{c}}$. This works well for large fields; however, the recommendation by AAPM (Ref. 3) to use caps in the measurement of $\mathrm{S}_{\mathrm{c}}$ can introduce errors as large as $10 \%$ for fields smaller than $10 \mathrm{~mm}$ width (Fig. 2). This study has validated an accurate method of measuring $S_{c}$ in small fields using tops instead of caps, establishing their use in routine clinical measurement.

\section{CONCLUSION}

This study identifies that the miniphantom design is the most important variable for reproducible and accurate measurements of the in-air output ratio, $S_{c}$, in small photon fields (less than $30 \mathrm{~mm}$ ). Three key features of the miniphantom design that need to be considered are: sidewall thickness, height above the detector, and overall width. Our results show 
that miniphantom sidewalls are not necessary for small fields, since most of the dose deposited is from the primary beam and not incident laterally on the detector. Furthermore, sidewalls can lead to significant errors in the determination of $\mathrm{S}_{\mathrm{c}}$ when the field becomes smaller than the physical size of the miniphantom. Whilst the height of the miniphantom had a negligible effect on $S_{c}$, tall narrow miniphantoms are difficult to position and are susceptible to alignment uncertainties. Therefore, we recommend using the minimum height necessary for removal of electron contamination. The overall width of the miniphantom should be smaller than the high dose region of the field, but sufficiently wide to be positioned accurately and reproducibly. It is only necessary for the top to cover the diameter of the sensitive detector element and not the entire detector housing. The approach we describe enables reliable and accurate measurement of $S_{c}$ for the smallest fields in routine clinical use.

\section{ACKNOWLEDGMENTS}

The work of B.H. was supported by a Cancer Council Western Australia PhD Top Up Scholarship. The authors acknowledge Dr. Bradley Oborn for his help and guidance associated with the Geant4 modeling and Matt Sloan for the high precision construction of tops, caps, and specialized equipment to enable the measurements to be made.

a) Author to whom correspondence should be addressed. Electronic mail: kirbie.warrener@sesiahs.health.nsw.gov.au; Telephone: 0449252644.

${ }^{1}$ M. M. Aspradakis, J. P. Byrne, H. Palmans, J. Conway, K. Rosser, J. Warrington, Report Number 103: Small Field MV Photon Dosimetry, edited by M. M. Aspradakis (IPEM, York, 2010).

${ }^{2}$ F. M. Khan, The Physics of Radiation Therapy, 4th ed. (Lippincott Wiliams \& Wilkin, Baltimore, MD, 2010).

${ }^{3}$ T. C. Zhu, A. Ahnesjo, K. L. Lam, X. A. Li, C. M. Ma, J. R. Palta, M. B. Sharpe, B. Thomadsen, and R. C. Tailor, "Report of AAPM Therapy Physics Committee Task Group 74: In-air output ratio, Sc, for megavoltage photon beams," Med. Phys. 36(11), 5261-5291 (2009).

${ }^{4}$ T. C. Zhu and B. E. Bjarngard, "The head-scatter factor for small field sizes," Med. Phys. 21(1), 65-68 (1994).

${ }^{5}$ J. P. Byrne and G. D. Lambert, "Collimator scatter factors for small photon treatment fields," in 8th Biennial ESTRO Meeting, Lisbon, Portugal, 2005.

${ }^{6} \mathrm{C}$. McKerracher and D. I. Thwaites, "Head scatter factors for small MV photon fields. Part II: The effects of source size and detector," Radiother. Oncol. 85(2), 286-291 (2007).

${ }^{7}$ A. J. Scott, A. E. Nahum, and J. D. Fenwick, "Monte Carlo modeling of small photon fields: Quantifying the impact of focal spot size on source occlusion and output factors, and exploring miniphantom design for smallfield measurements," Med. Phys. 36(7), 3132-3144 (2009).

${ }^{8}$ C. McKerracher and D. I. Thwaites, "Head scatter factors for small MV photon fields. Part I: A comparison of phantom types and methodologies," Radiother. Oncol. 85(2), 277-285 (2007).

${ }^{9}$ S. Dieterich and G. W. Sherouse, "Experimental comparison of seven commercial dosimetry diodes for measurements of stereotactic radiosurgery cone factors," Med. Phys. 38(7), 4166-4173 (2011).

${ }^{10}$ B. R. Thomadsen, S. S. Kubsad, B. R. Paliwal, S. Shahabi, and T. R. Mackie, "On the cause of the variation in tissue-maximum ratio values with source-to-detector distance," Med. Phys. 20(3), 723-727 (1993).
${ }^{11}$ X. A. Li, M. Soubra, J. Szanto, and L. H. Gerig, "Lateral electron equilibrium and electron contamination in measurements of head-scatter factors using miniphantoms and brass caps," Med. Phys. 22(7), 1167-1170 (1995).

${ }^{12} \mathrm{P}$. A. Jursinic, "Measurement of head scatter factors of linear accelerators with columnar miniphantoms," Med. Phys. 33(6), 1720-1728 (2006).

${ }^{13}$ J. Lambert, Y. Yin, D. R. McKenzie, S. H. Law, A. Ralston, and N. Suchowerska, "A prototype scintillation dosimeter customized for small and dynamic megavoltage radiation fields," Phys. Med. Biol. 55(4), 1115-1126 (2010).

${ }^{14}$ D. C. Konnoff, T. K. Plant, and E. Shiner, "SSPM base radiation sensing: Preliminary laboratory and clinical results," Radiat. Meas. 46(1), 76-87 (2011).

${ }^{15}$ P. Z. Liu, N. Suchowerska, J. Lambert, P. Abolfathi, and D. R. McKenzie, "Plastic scintillation dosimetry: Comparison of three solutions for the Cerenkov challenge," Phys. Med. Biol. 56(18), 5805-5821 (2011).

${ }^{16}$ P. Z. Liu, N. Suchowerska, P. Abolfathi, and D. R. McKenzie, "Real-time scintillation array dosimetry for radiotherapy: The advantages of photomultiplier detectors," Med. Phys. 39(4), 1688-1695 (2012).

${ }^{17}$ T. Aland, T. Kairn, and J. Kenny, "Evaluation of a Gafchromic EBT2 film dosimetry system for radiotherapy quality assurance," Australas. Phys. Eng. Sci. Med. 34(2), 251-260 (2011).

${ }^{18}$ M. A. Stevens, J. R. Turner, R. P. Hugtenburg, and P. H. Butler, "Highresolution dosimetry using radiochromic film and a document scanner," Phys. Med. Biol. 41(11), 2357-2365 (1996).

${ }^{19}$ D. W. O. Rogers, B. Walters, and I. Kawrakow, BEAMnrc Users Manual (NRC, Canada, 2011).

${ }^{20}$ D. W. Rogers, B. A. Faddegon, G. X. Ding, C. M. Ma, J. We, and T. R. Mackie, "BEAM: A Monte Carlo code to simulate radiotherapy treatment units," Med. Phys. 22(5), 503-524 (1995).

${ }^{21}$ B. Walters, I. Kawrakow, and D. W. O. Rogers, DOSXYZnrc Users Manual (National Research Council of Canada, Ottawa, 2011).

${ }^{22}$ S. Agostinelli, J. Allison, K. Amako, J. Apostolakis, H. Araujo, P. Arce, M. Asai, D. Axen, S. Banerjee, G. Barrand, F. Behner, L. Ballagamba, J. Boudreau, L. Broglia, A. Brunengo, H. Burkhardt, S. Chauvie, J. Chuma, R. Chytracek, G. Cooperman, G. Cosmo, P. Degtyarenko, A. Dell'Acqua, G. Depaola, D. Dieterich, R. Enami, A. Feliciello, C. Ferguson, H. Fesefeldt, G. Folger, F. Foppiano, A. Forti, S. Garelli, S. Gianoi, R. Giannitrapani, D. Gibin, J. J. Gomez Cadenas, I. Gonzalez, G. Gracia Abril, G. Greeniaus, W. Greiner, V. Grichine, A. Grossheim, S. Guatelli, P. Gumplinger, R. Hamatsu, K. Hashimoto, H. Hasui, A. Heikkinen, A. Howard, V. Ivanchenko, A. Johnson, F. W. Jones, J. Kallenbach, N. Kanaya, M. Kawabata, M. Kawaguti, S. Kelner, P. Kent, A. Kimura, T. Kodama, R. Kokoulin, M. Kossov, H. Kurashige, E. Lamanna, T. Lampen, V. Lara, V. Lefebure, F. Lei, M. Liendl, W. Lockman, F. Longo, S. Magni, M. Maire, E. Medernach, K. Minamimoto, P. Mora de Freitas, Y. Morita, K. Murakami, M. Nagamatu, R. Nartallo, P. Nieminen, T. Nishimura, K. Ohtsubo, M. Okamura, S. O’Neale, Y. Oohata, K. Paech, J. Perl, A. Pfeiffer, M. G. Pia, F. Ranjard, A. Rybin, S. Sadilov, E. Di Salvo, G. Santin, T. Sasaki, N. Savvas, Y. Sawada, S. Scherer, S. Sei, V. Sirotenko, D. Smith, N. Starkov, H. Stoecker, J. Sulkimo, M. Takahata, S. Tanaka, E. Tcherniaev, E. Safai Tehrani, M. Tropeano, P. Truscott, H. Uno, L. Urban, P. Urban, M. Verderi, A. Walkden, W. Wander, H. Weber, J. P. Wellisch, T. Wenaus, D. C. Williams, D. Wright, T. Yamada, H. Yoshida, and D. Zschiesche, "GEANT4 - A simulation toolkit," Nucl. Instrum. Methods Phys. Res. A 506(3), 250-303 (2003).

${ }^{23}$ P. Andreo, D. T. Burns, K. Hohlfeld, M. S. Huq, T. Kanai, F. Laitano, V. Smyth, and S. Vynckier, Absorbed Dose Determination in External Beam Radiotherapy: An International Code of Practice for Dosimetry based on Standards of Absorbed Dose to Water (IAEA, Vienna, 2000).

${ }^{24}$ A. J. Scott, S. Kumar, A. E. Nahum, and J. D. Fenwick, "Characterizing the influence of detector density on dosimeter response in non-equilibrium small photon fields," Phys. Med. Biol. 57(14), 4461-4476 (2012).

${ }^{25}$ A. Ralston, P. Liu, K. Warrener, D. McKenzie, and N. Suchowerska, "Small field diode correction factors derived using an air core fibre optic scintillation dosimeter and EBT2 film," Phys. Med. Biol. 57(9), 2587-602 (2012). 